\title{
A cookbook for DNase Hi-C
}

\author{
Maria Gridina', Evgeniy Mozheiko', Emil Valeev ${ }^{1,2}$, Ludmila P. Nazarenko ${ }^{3}$, Maria E. Lopatkina ${ }^{3}$, \\ Zhanna G. Markova ${ }^{5}$, Maria I. Yablonskaya ${ }^{4}$, Viktoria Yu Voinova ${ }^{4}$, Nadezhda V. Shilova ${ }^{5}$, Igor N. Lebedev ${ }^{3}$ and \\ Veniamin Fishman ${ }^{1,2^{*}}$ (1)
}

\begin{abstract}
Background: The Hi-C technique is widely employed to study the 3-dimensional chromatin architecture and to assemble genomes. The conventional in situ $\mathrm{Hi}-\mathrm{C}$ protocol employs restriction enzymes to digest chromatin, which results in nonuniform genomic coverage. Using sequence-agnostic restriction enzymes, such as DNAse I, could help to overcome this limitation.
\end{abstract}

Results: In this study, we compare different DNAse Hi-C protocols and identify the critical steps that significantly affect the efficiency of the protocol. In particular, we show that the SDS quenching strategy strongly affects subsequent chromatin digestion. The presence of biotinylated oligonucleotide adapters may lead to ligase reaction by-products, which can be avoided by rational design of the adapter sequences. Moreover, the use of nucleotideexchange enzymes for biotin fill-in enables simultaneous labelling and repair of DNA ends, similar to the conventional $\mathrm{Hi}-\mathrm{C}$ protocol. These improvements simplify the protocol, making it less expensive and time-consuming.

Conclusions: We propose a new robust protocol for the preparation of DNAse Hi-C libraries from cultured human cells and blood samples supplemented with experimental controls and computational tools for the evaluation of library quality.

Keywords: DNAse I, Hi-C, Genome organization, Human peripheral blood, K562, LNCaP, A549

\section{Background}

The coupling of the chromatin conformation capture technique with next-generation sequencing has resulted in the development of a simple and efficient Hi-C protocol, which enables the genome-wide chromatin architecture to be studied $[1,2]$. Along with numerous insights into nuclear organization and dynamics, $\mathrm{Hi}-\mathrm{C}$ results have demonstrated that spatial contacts between loci strongly depend on the genomic distance between them $[1,2]$. In particular, adjacent genomic segments interact considerably more frequently than distal or interchromosomal regions. This dependence of chromatin contacts on genomic distance has been observed in all studied

${ }^{*}$ Correspondence: minja-f@ya.ru

${ }^{1}$ Institute of Cytology and Genetics SB RAS, Lavrentjeva ave 10, Novosibirsk, Russia

Full list of author information is available at the end of the article cell types [3-5] and can be utilized to infer the order of scaffolds in poorly assembled genomes, providing chromosome-length assemblies [6-11]. For species with a well-assembled genome, such as humans, the Hi-C technique can be used to detect structural variations, which alter the order of genomic segments and therefore lead to significant changes in chromatin interaction frequencies [6, 12-17]. In addition, one can extract information about single nucleotide variations (SNVs) from $\mathrm{Hi}-\mathrm{C}$ reads. The studies described in $[18,19]$ have shown that coupling proximity information and SNV data can be used to phase genomes, and we have recently suggested using a cognate approach for genetic diagnostics [20].

Classical Hi-C protocols rely on restriction enzymes for fragmenting genomic DNA [1, 2]. This fragmentation limits the theoretical resolution of $\mathrm{Hi}-\mathrm{C}$ analysis by the restriction fragment length and results in nonuniform genomic coverage biased towards the regions flanking the 
restriction enzyme recognition sites. For most genomewide analyses of chromatin architecture, this limitation is not essential because achieving a resolution beyond the restriction fragment length would require an extremely high sequencing depth. However, for capture-Hi-C data, as well as for scaffolding or genotyping applications, high resolution and uniform coverage are desirable.

Several solutions have been proposed to overcome these limitations. First, 4-cutter enzymes are currently used to prepare Hi-C libraries, which decreases the average fragment length compared to the 6-cutter enzymes used previously [2]. Moreover, a combination of several restriction enzymes can be utilized to decrease fragment length. However, the distribution of cut sites in these cases is not uniform, and there are always some genomic regions that are not well represented in a $\mathrm{Hi}-\mathrm{C}$ dataset prepared using restriction enzymes.

Second, nucleases that have no sequence-specific cutting preferences, such as DNase I [21-23] or MNase $[24,25]$, can be used in the Hi-C protocol. MNase has recently been utilized to prepare high-resolution wholegenome Hi-C datasets of yeasts and humans $[24,25]$. At the same time, the Zhijun Duan group has developed a protocol for the preparation of capture- and genomewide $\mathrm{Hi}-\mathrm{C}$ datasets using DNase I [21-23]. Providing uniform coverage and a theoretically unlimited resolution of data, these protocols seem to be the most suitable when using $\mathrm{Hi}-\mathrm{C}$ sequencing for genotyping purposes.

In this study, we aimed to optimize the DNase Hi-C protocol to allow efficient capture of chromatin interactions in human cells. We showed that optimization of the cell lysis and chromatin digestion conditions by DNase I was critical for the preparation of high-quality libraries. We also compared two different strategies for DNA endlabelling: the original strategy employing biotinylated linkers and an alternative strategy relying on nucleotide exchange. Based on our observations, we suggested several controls that enabled us to estimate library quality before and after sequencing. Finally, we showed that Burrows-Wheeler Aligner could efficiently map chimaeric reads produced both in the presence and absence of biotinylated linkers and provided bioinformatic tools and pipelines suitable for the analysis of $\mathrm{DNase} \mathrm{Hi}-\mathrm{C}$ data.

\section{Results}

We started our study by benchmarking the published DNAse $\mathrm{Hi}-\mathrm{C}$ protocol developed by $\mathrm{Ma}$ et al. [22]. For this benchmarking, we reanalysed published data and applied the protocol without modifications to a collection of human blood samples and K562 cells. Through the manuscript, we label the reanalysis of the original data as "Ma et al. (reanalysed)", whereas new assays following the original protocol are labelled as "Protocol: Ma et al." We follow the same naming conventions for another protocol published by Ramani et al. [21], which we also benchmarked. When referring to the modified versions of these protocols developed in this study, we highlight key modifications in the protocol title, for example, "Protocol: Ramani et al. long linker". We list all the protocols used in this study in Table 1.

Our first attempts to use the DNase $\mathrm{Hi}-\mathrm{C}$ protocol by $\mathrm{Ma}$ et al. resulted in low-quality libraries (Fig. 1a, b; Additional file 1: Table S1). In particular, we found a large proportion of interchromosomal (trans) interactions, indicating high-level random ligations. There was also a large excess of read pairs in the inward (forward-reverse) orientation (also called "dangling ends" or DEnds), suggesting low digestion and/or ligation efficiency. Moreover, there were overrepresented sequences corresponding to ligation products between the oligonucleotide adapters used for DNA end-labelling. Finally, a high percentage of reads failed to align due to the presence of adapter

Table 1 DNAse Hi-C protocols used in this study

\begin{tabular}{ll}
\hline Protocol name & Short protocol description \\
\hline $\begin{array}{l}\text { Ma et al. (reanalysed) } \\
\text { Ramani et al. (reanalysed) }\end{array}$ & $\begin{array}{l}\text { Data from Ma et al. [22] reanalysed using our pipeline } \\
\text { Protocol Ma et al. }\end{array}$ \\
$\begin{array}{l}\text { Reproduction of the protocol from [22] in our laboratory } \\
\text { Protocol Ma et al. new blunt }\end{array}$ & $\begin{array}{l}\text { Modification of the protocol from [22]. We changed the blunt-adapter sequences to prevent the formation of } \\
\text { adapter multimers }\end{array}$ \\
Protocol Ma et al. long linker & $\begin{array}{l}\text { Modification of the protocol from [22]. We abolished the two-step ligation procedure. We used the long linker } \\
\text { derived from the BAT-Hi-C protocol instead }\end{array}$ \\
$\begin{array}{l}\text { Protocol Ramani et al } \\
\text { Protocol Ramani et al. long linker }\end{array}$ & $\begin{array}{c}\text { Modification of the protocol from [21]. We abolished the two-step ligation procedure and used the long linker } \\
\text { derived from the BAT-Hi-C protocol instead }\end{array}$ \\
Protocol Ramani et al. biotin fill-in & $\begin{array}{c}\text { Modification of the protocol from [21]. We abolished the two-step ligation procedure. End-labelling with bioti- } \\
\text { nylated dCTP nucleotides was performed during the end-repair step following DNase treatment }\end{array}$ \\
\hline
\end{tabular}



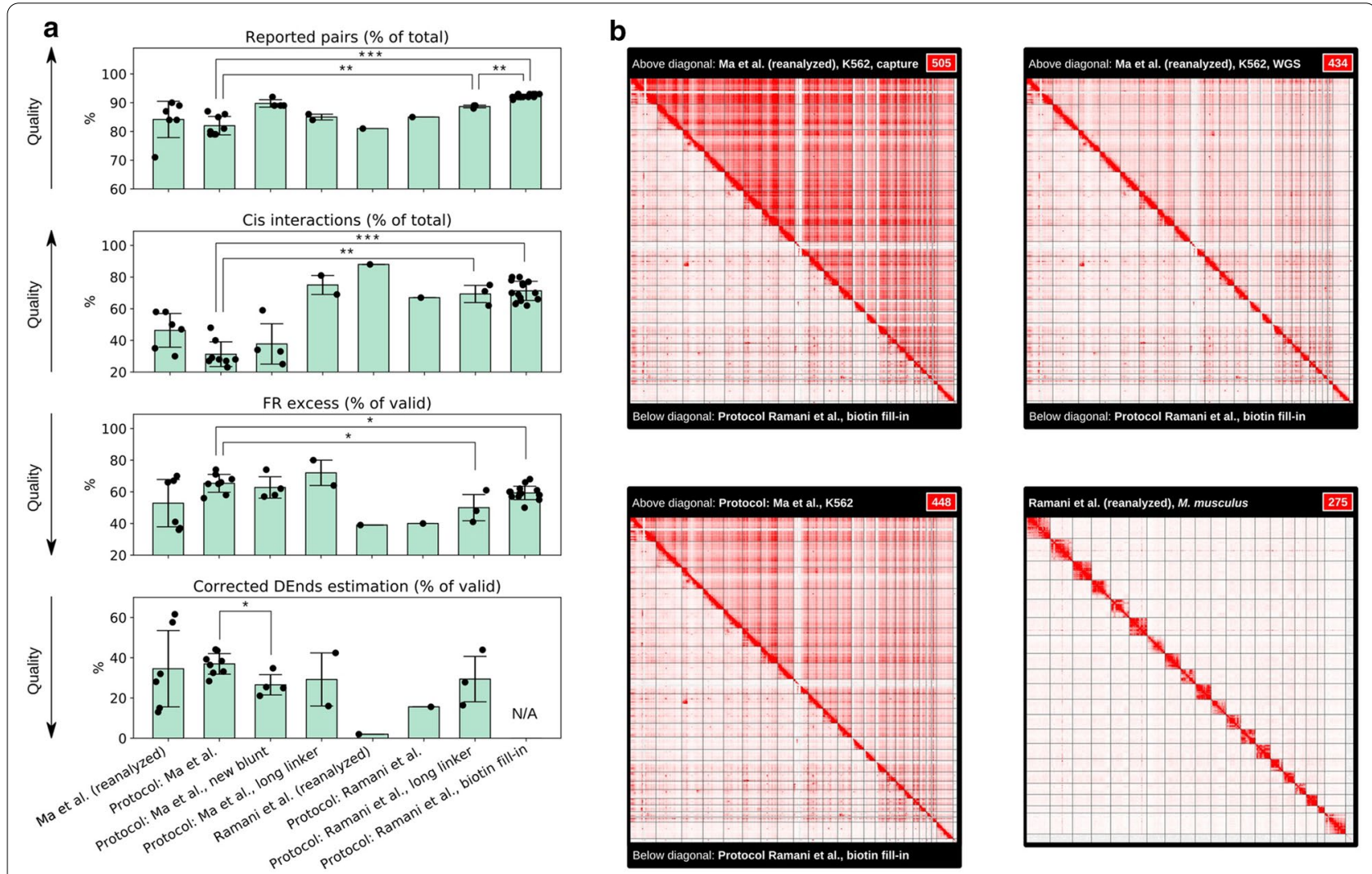

Fig. 1 Improving the DNAse Hi-C protocol allows the generation of high-quality Hi-C maps. a Quality metrics of Hi-C datasets. Data are grouped according to the protocol employed for library construction. Each dot represents an independent $\mathrm{Hi}-\mathrm{C}$ library preparation. The dataset names are explained in Table 1, and all the details of each protocol are described in the "Methods" section. Note that Ramani et al. ([21]) performed Hi-C on mouse samples, whereas other data were from human cells, which could explain some of the differences between "Ramani et al. (reanalysed)" and other samples. The reported pairs percentage indicates the mapping efficiency; cis-interactions reflect noise levels; FR-excess indicates the overrepresentation of reads in the forward-reverse (inward) orientation, a signature of undigested or unligated DNA (DEnds); when possible, estimated DEnds were corrected using information about biotinylated linker incorporation (see "Methods" section) and are shown on the corrected DEnds estimation plot. The significance of differences between groups was estimated using the Mann-Whitney test. $\mathbf{b}$ Representative Hi-C data obtained using different protocols for human $\mathrm{K} 562$ cells and for mouse brain cells (in the case of reanalysed Ramani et al. data). For K562 cells, each $\mathrm{Hi}$-C heatmap shows a comparison between results obtained using the protocol from [23] (above the diagonal line) and data obtained using the biotin fill-in protocol developed in this study (below the diagonal line). All data were downsampled to the same sequencing depth, and the number in the top right corner indicates the values selected on the Juicebox colour slider. Additional heatmaps showing representative genomic regions are shown in Additional file 1: Fig. AS1

multimers. To optimize the protocol, we prepared and sequenced a few dozen DNase Hi-C libraries. By trial and error, we identified several critical steps that significantly affected the efficiency of the protocol (see Fig. 1a for a comparison of the protocol quality metrics and Fig. 1b and Additional file 1: Fig. S1 for representative Hi-C maps). We summarized our experience as a set of hints and quality controls supplemented with a detailed proto$\mathrm{col}$ and representative results.

\section{Hint 1. Digest the chromatin properly Problem}

Our initial attempts to digest cross-linked chromatin using DNAse I showed that this step was not easy to reproduce. The distribution of fragment lengths obtained after digestion varied from sample to sample (Fig. 2a). Moreover, even at high enzyme concentrations, when the median fragment length was approximately $100 \mathrm{bp}$, a detectable amount of undigested high-molecular weight DNA was present in the reaction (as shown in Fig. 2a, lanes 4 and 5). Notably, our results showed that highquality $\mathrm{Hi}-\mathrm{C}$ libraries could not be obtained in this case (see the metrics of Protocol: Ma et al. in Fig. 1).

\section{Solution}

Hi-C protocols include a nuclear permeabilization step, which allows subsequent enzymatic digestion of chromatin. This digestion is typically achieved by an SDS 
a

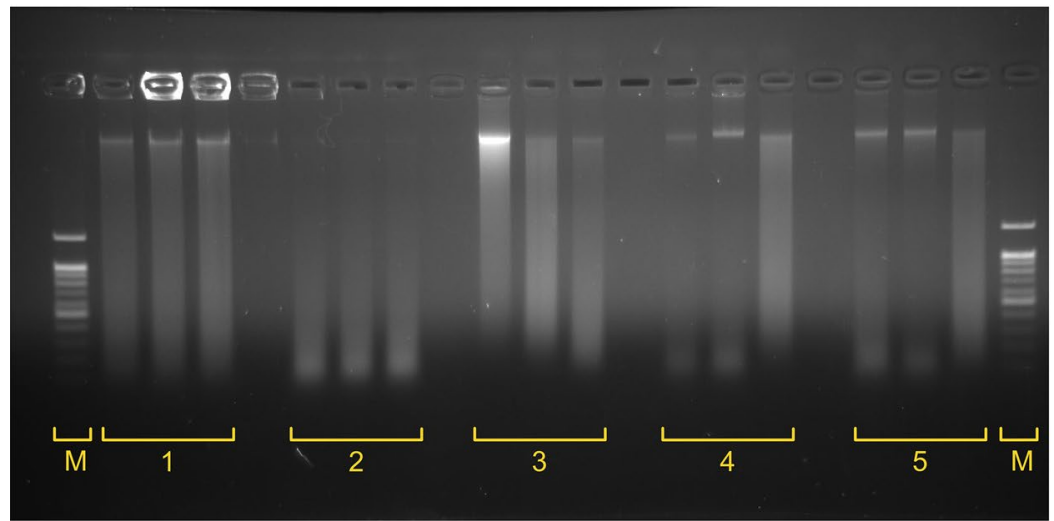

b

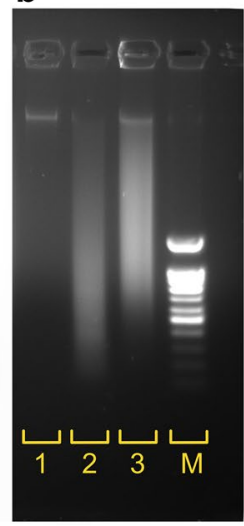

Fig. 2 Chromatin digestion and ligation in DNAse Hi-C experiments. a) Reproducibility of chromatin digestion by DNase I under the conditions recommended by Ramani et al. [21] and Ma et al. [22] assessed in three independent replicas. 1—Ramani et al., 1 U DNase I. 2 -Ramani et al., 2 U DNase I. 3-Ma et al., 2 U DNase I. 4-Ma et al., 4 U DNase I. 5-Ma et al., 6 U DNase I. Lane M shows a 100 bp DNA ladder. b Intermediate controls of high-quality libraries: 1 -intact gDNA, 2-DNase I digestion of cross-linked chromatin under the conditions described by Ramani et al., and 3ligation of DNase I-digested chromatin from lane 2

treatment in the presence of EDTA. However, DNase I is highly sensitive to the presence of metal chelators and SDS. To allow DNase I digestion after the permeabilization step, Ma et al. [22] suggested removing SDS and EDTA by sedimenting and washing chromatin. Alternatively, the DNase Hi-C protocol published by Ramani and colleagues [21] suggested not using EDTA and sequestering SDS with Triton X-100, similar to many classical Hi-C protocols. We aimed to compare the protocols of $\mathrm{Ma}$ et al., Ramani et al. and their modifications. As shown in Figs. 1a and 2a, in almost all cases, the protocol suggested by Ramani et al. resulted in:

1. A more reproducible DNase I digestion pattern with fragment lengths distributed between 100 and 1000 bp (a representative example is shown in Fig. 2 B, lane 2);

2. Higher overall protocol yields, and

3. A significantly lower noise ratio (measured as the percentage of cis-interactions).

We noted that the yield of DNA after biotin pulldown was a very good indicator of library quality. For example, libraries obtained without SDS quenching required more cycles of amplification after pulldown and resulted in a lower yield. In addition, analysis of sequencing reads with FastContext, a bioinformatic tool that we developed to assist with DNase $\mathrm{Hi}-\mathrm{C}$ data analysis, showed that a large portion of reads from these low-quality libraries did not contain biotinylated adapters (Additional file 1: Fig. 2A), whereas the percentage of adapter-containing reads was higher for high-quality libraries (Additional file 1:
Fig. S2B). Thus, libraries obtained without SDS quenching mostly represented undigested or unligated DNA.

\section{Quality controls}

1. Always check digestion and ligation of the chromatin using gel electrophoresis. Representative results are shown in Fig. 2b, lanes 2-3.

2. Estimate the streptavidin pulldown efficiency and compare the pulldown yield with the unlabelled DNA control. Streptavidin pulldown of $1 \mu \mathrm{g}$ of biotin-unlabelled genomic DNA followed by 12 cycles of PCR yielded $\sim 30-42 \mathrm{ng}$ (average $=36.2 \mathrm{ng}, n=4$ ) of product. In contrast, we obtained at least $500 \mathrm{ng}$ DNA from 6 cycles of PCR for successful library generation. This pulldown yield was similar when biotin labelling was performed using ligated biotinylated adapters or nucleotide exchange (see below). We found that it was critical to obtain several times more product after pulldown of libraries than in control reactions performed with the same amount of unlabelled DNA.

\section{Hint 2. Do not use unnecessary ingredients: excluding biotinylated adapters simplifies library preparation and data analysis \\ Problem}

In classical $\mathrm{Hi}-\mathrm{C}$ and Micro- $\mathrm{C}$ protocols, the end-repair reaction with biotinylated nucleotides follows a digestion step, which allows DNA end-labelling and subsequent selection of ligation junctions. In the published DNAse 
$\mathrm{Hi}-\mathrm{C}$ protocols, end-labelling is achieved via ligation of biotinylated oligonucleotides (adapters). Because ligation of adapters requires a sticky A-end, this reaction depends on the efficiency of A-tailing. In the recently published protocol by $\mathrm{Ma}$ et al., the authors additionally use a blunt adapter ligated to DNA ends if these ends that skip A-tailing. Adapter ligation introduces extra steps in the protocol, making it more complicated and less efficient.

Analysis of the data produced by Ma et al., as well as our own data, showed that oligonucleotide adapters were not only ligated to the DNA ends but also to each other, forming dimers and multimers (highlighted in yellow in Additional file 1: Fig. S2a). We illustrate in Fig. 3a that these undesired ligations between adapters can block proximity ligation, thereby leading to the low overall efficiency of the protocol and the increase of dangling ends and spurious interactions. Moreover, ligation of adapters made $\mathrm{Hi}-\mathrm{C}$ data analysis more complicated, requiring the detection of adapter sequences and various ligation products between adapters in $\mathrm{Hi}-\mathrm{C}$ reads before mapping (this problem will be additionally discussed below).

\section{Solution}

We showed that the formation of adapter multimers was due to the sequence similarity of the blunt and bridge adapters (Fig. 3a) and could be reproduced in a control ligation reaction (Fig. 3b). Changing the adapter sequences prevented the formation of adapter multimers (Fig. 3b), which led to the reduction of undigested/ unligated fragments (see the corrected DEnds fraction metrics in Fig. 1a for the protocol "Ma et al., new blunt"). Additionally, we showed that the single adapter derived from the BAT-Hi-C protocol could be used as a substitute for the two-part bridge oligonucleotides. Using the single adapter allowed the generation of high-quality libraries (see the metrics in Fig. 1, "Protocol: Ramani et al., long linker" and "Protocol: Ma et al., long linker") without using blunt adapters and a two-step ligation procedure, simplifying the experimental and computational steps.

We next decided to avoid the usage of any biotinylated oligonucleotide adapters. To achieve this aim, we performed end-labelling with biotinylated dCTP nucleotides during the end-repair step following DNase treatment. To the best of our knowledge, this report describes the first time the DNase $\mathrm{Hi}-\mathrm{C}$ protocol has been performed without biotinylated adapters. We found that this simplified protocol allowed us to generate high-quality data (Fig. 1a, "Protocol: Ramani et al.+biotin fill-in"). Thus, we recommend using no adapters and employing a biotin fill-in strategy to prepare DNase Hi-C libraries.

\section{Quality control}

1. If adapters were used for end-labelling, then a ligation assay was performed to ensure that the adapters could not form multimers during library preparation. Representative results are shown in Fig. 3b.

2. When processing data, we recommend searching for adapter multimers in read sequences. To achieve this aim, we developed the bioinformatic tool FastContext, which reports the relative abundance of different adapters and their combinations in sequenced reads. Representative results are shown in Additional file 1: Fig. S2.

\section{Hint 3. Do not treat dangling ends as artefacts Problem}

We observed a large number of "dangling ends" fragments in the DNase Hi-C libraries. We quantified dangling ends as excess reads in the inward (forward-reverse, FR) orientation over reads in the same (forward-forward, FF or reverse-reverse, RR) orientation. This excess showed strong dependence on distance and sharply dropped when the distance between reads exceeded $1 \mathrm{~kb}$. Excess FR reads are found in virtually all $\mathrm{Hi}-\mathrm{C}$ libraries, and such reads are usually interpreted as representations of undigested or unligated DNA.

\section{Solution}

We hypothesized that the large excess of FR reads might be due to frequent back-ligation events when the DNA

\footnotetext{
(See figure on next page.)

Fig. 3 Using biotinylated adapters complicates the DNAse $\mathrm{Hi}$-C protocol and results in undesired by-products. a Schematic description of possible ligation reaction products obtained in the presence of biotinylated adapters from Ma et al. [22]. The adapters used by Ma et al. are referred to as bridge and blunt oligonucleotides. As evident from the scheme, sequence similarity between bridge and blunt adapters leads to the formation of multimers. $\mathbf{b}$ Ligation assays showing the formation of adapter multimers. Lanes 1 and 10-100-bp ladder. Lanes 2 and 3-bridge (2) and blunt (3) oligonucleotides. Lanes 4-6-self-ligation assays performed with bridge (4), blunt (5), and bridge/blunt oligonucleotides mix (6). Lanes 7-9-bridge (7), blunt (8), and bridge/blunt oligonucleotides mix (9) subjected to ligation, followed by phosphorylation and an additional round of ligation, which imitated enzymatic steps during the DNAse Hi-C protocol. The arrow shows the adapter multimerization products. Lane $11-$ self-ligation assay of the redesigned blunt adapter, which lacks sequence similarity with the bridge adapter. Lane 12 —redesigned blunt adapter subjected to ligation, phosphorylation and an additional round of ligation. Lanes 13 and 14-the same reactions as in lanes 11 and 12 performed using a mix of bridge and redesigned blunt-adapter oligonucleotides
} 
a

Expected reactions

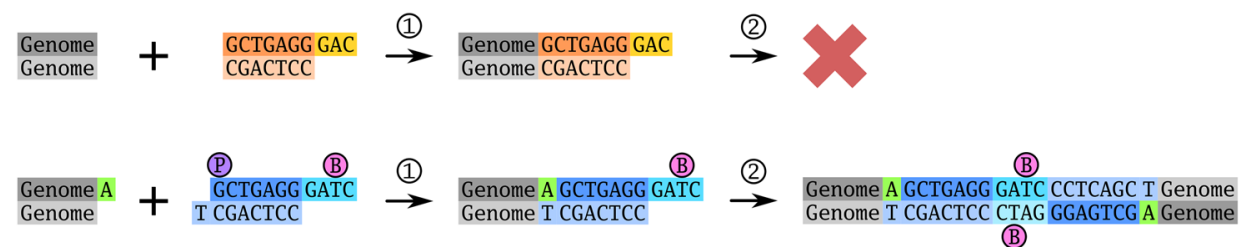

Unwanted reaction: Strand switch \& chimeric adapter ligation

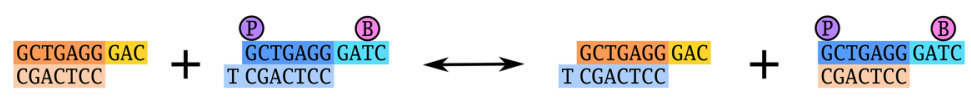

$\underset{\text { Genome A }}{\text { Genome }}+\underset{\text { I GGACTCC }}{\text { GCTGAGG GAC }} \stackrel{\stackrel{(1)}{\rightarrow}}{\rightarrow} \underset{\text { Genome I CGACTCC }}{\text { Genom ACTGGG }} \stackrel{(2)}{\rightarrow}$

Unwanted reaction: Chimeric adapter concatemerization
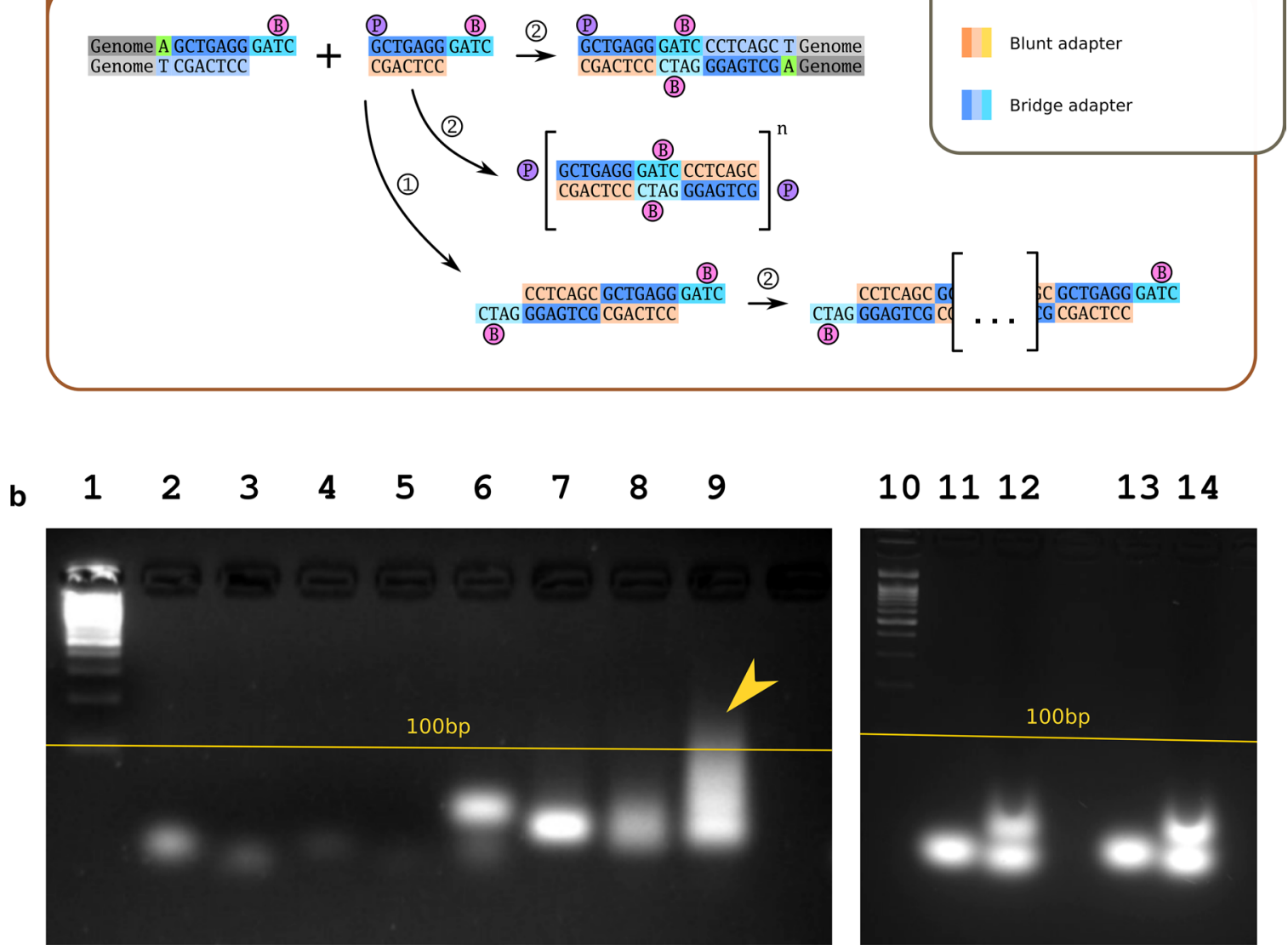
ends were preferentially joined in the same order as in the intact (undigested) genome during the ligation step. We were able to assess the frequency of back ligations by analysing DNase $\mathrm{Hi}-\mathrm{C}$ libraries prepared in the presence of biotinylated oligonucleotide adapters. The adapters marked ligation junctions; therefore, all reads in the FR orientation harbouring the adapter represented back-ligation events, rather than undigested or unligated chromatin. However, not all ligation junctions incorporated bridge adapters, some DNA ends could be ligated directly. To correctly account for this factor, we estimated the probability of adapter-free ligation events from the number of adapter-free interchromosomal read pairs, which represent all ligation events ("Methods" section). This approach allowed us to compute, for the first time, the frequency of back-ligation events in $\mathrm{Hi}-\mathrm{C}$ libraries.

Notably, up to $75 \%$ of the excess FR reads were explained by back-ligation events (Fig. 1a; Additional file 1: Table S1). This result suggests that after digestion, DNA ends were preferentially located in close proximity to each other, which promoted back-ligation.

\section{Quality control}

The high fraction of dangling ends does not necessarily reflect low digestion/ligation efficiency and should not be used as a quality control. Instead, the ratio of intra- to interchromosomal interactions reflects library quality. This metric can be computed using the computational tools described in the manuscript or other software [26].

\section{Hint 4. Burrows-Wheeler Aligner allows the analysis of $\mathrm{Hi}-\mathrm{C}$ data without knowing the ligation junction motif Problem}

Many $\mathrm{Hi}-\mathrm{C}$ reads are chimaeric, i.e. they contain junctions between different genomic fragments. This feature might affect the mappability of $\mathrm{Hi}-\mathrm{C}$ reads. When using restriction enzymes to digest chromatin, a specific ligation junction site allows chimaeric read splitting. A similar approach can be employed when using bridge adapters in the DNAse Hi-C protocol. However, we observed that using adapters reduced $\mathrm{Hi}-\mathrm{C}$ read mappability (as evident from the number of reported pairs in Fig. 1a, Additional file 1: Table S1, and Fig. 4a and b), and splitting chimaeric reads containing expected adapter junction sequences could not fully address this problem (Additional file 1 : Table S2, Fig. 4a). Analysis of unaligned reads showed that they contained adapter multimers, both at the read ends and in the middle of the fragments. This finding was in line with our observations of adapter multimerization. Removing these adapter sequences improved the alignment (Fig. 4a), but required sophisticated bioinformatic pipelines ("Methods" section). Finally, for libraries

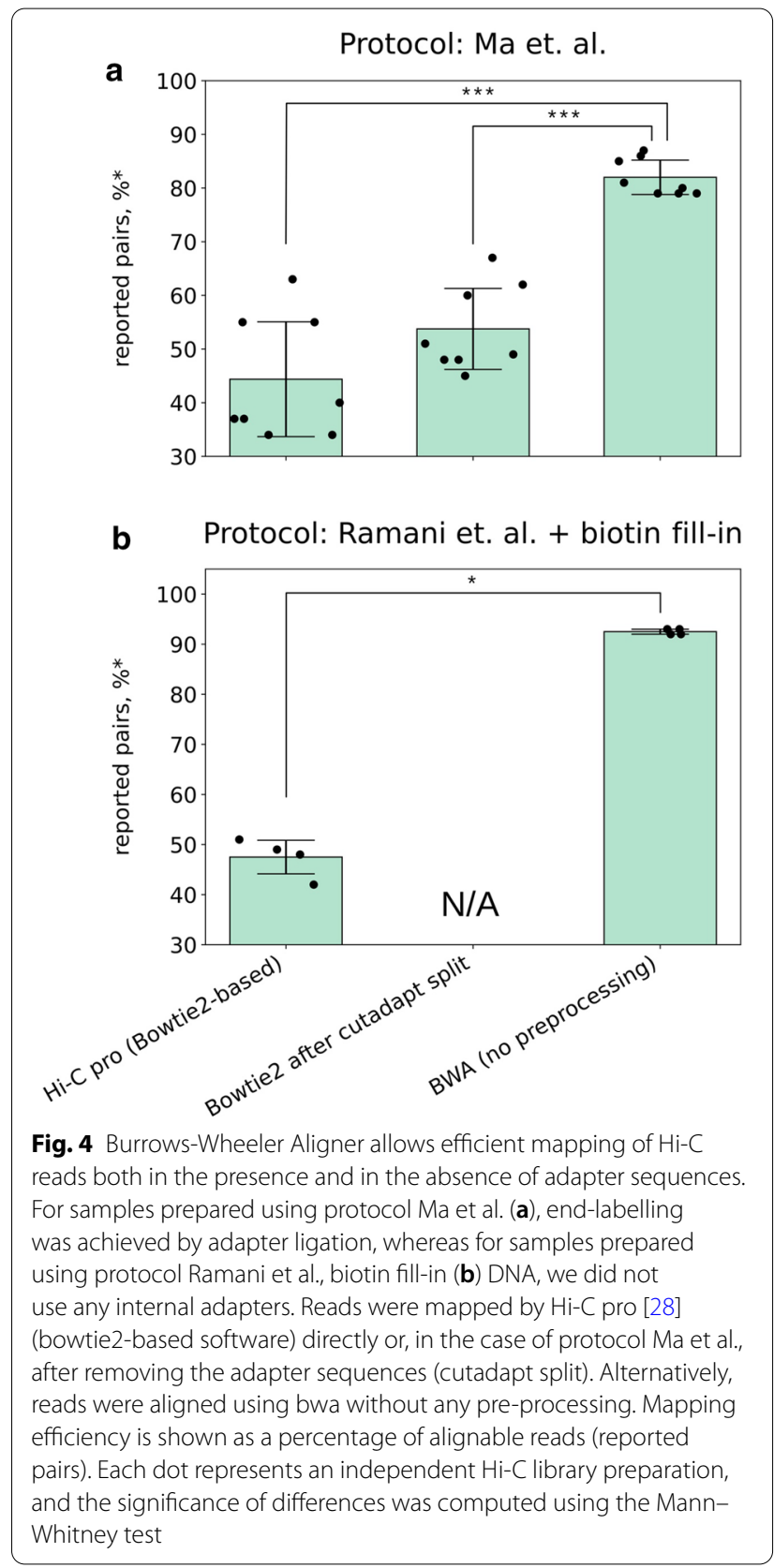

prepared without bridge adapters, as suggested above, it is impossible to find ligation junctions before alignment.

\section{Solution}

We found that Burrows-Wheeler Aligner (bwa) [27] could efficiently align chimaeric reads (Fig. 4b). For reads containing adapter multimers, reads split (or trimmed) using expected adapter sequences, and reads obtained from DNAse $\mathrm{Hi}-\mathrm{C}$ libraries without adapters, bwa showed significantly better results than another commonly used aligner, bowtie2 (Additional file 1: Table S2, 
Fig. 4b). Thus, we recommend using bwa for $\mathrm{Hi}-\mathrm{C}$ data processing and note that the adapter trimming step is not necessary.

\section{Quality control}

For human data, approximately $90 \%$ of pairs could be mapped unambiguously at both ends when using bwa for mapping. The mapping statistics can be accessed using the pipeline accompanying the manuscript.

\section{Discussion}

DNAse $\mathrm{Hi}-\mathrm{C}$ methods are used relatively rarely, most likely due to their complicated experimental design, which includes optimization of the chromatin digestion conditions and the use of biotinylated adapters. Our results showed that the DNAse I digestion step could be reproducible when using optimal cell lysis and SDS quenching conditions, as suggested in [21]. Furthermore, we showed that the use of biotinylated adapters was not necessary and that biotin could be incorporated by applying a fill-in strategy. Although the fill-in and adapterbased strategies both resulted in high-quality data when using optimal cell lysis and SDS quenching conditions, the former simplified the experimental protocol and prevented the formation of ligation by-products.

Finally, there was no need to identify the ligation junction sites within sequencing fragments because BurrowsWheeler Aligner could efficiently map chimaeric reads. Overall, our work shows that DNAse I Hi-C is a robust and efficient method that can be easily applied to study chromatin interactions in human cells.

Notably, we found that using a single biotinylated nucleotide (biotin-dCTP in our case) was sufficient for labelling DNA ends. This could be explained by several observations. First, DNAse I in the presence of Mn2 + introduces double-stranded breaks with $5^{\prime}$-overhangs with a length of 4 bp $[32,33]$. When filling this overhang, biotin-dCTP nucleotides are likely to be introduced.

Second, even if a fraction of DNA ends appear to be blunt, the Klenow enzyme has $3^{\prime}-5^{\prime}$ exonuclease activity, which enables the exchange of nucleotides at blunt ends. A previous study [34] suggests that not only terminal, but also internal nucleotides can be labelled due to this activity, which reduces the dependence of labelling on the sequence of the DNA end.

Finally, because both blunt- and cohesive-ends could be labelled and DNA breaks occur independently in each cell, it follows that labelling with biotinylated dCTP (or any other single biotinylated dNTP) could occur in almost any genomic region.

The main advantage of the DNAse I Hi-C method is that it enables more uniform coverage to be achieved than the conventional $\mathrm{Hi}-\mathrm{C}$ technique. However, we note that there are other approaches aimed at generating $\mathrm{Hi}-\mathrm{C}$ libraries with uniform coverage employing, for example, MNase to fragment the genome [24]. Moreover, DNase and MNase have their own smaller and larger sequencespecific biases, respectively, and using a combination of several 4-cutter restriction endonucleases (up to three in the Arima Genomics kit) could provide comparable, if not the same, coverage as DNAse I.

Analysis of the ligation junctions marked by biotinylated oligonucleotides showed that the orientation of DNA ends during the ligation step was not random. Our data demonstrated a strong preference for the ligation of DNA fragments in the same order as they occur in the intact genome. This preference is most likely due to tight formaldehyde fixation, which does not allow rotation or diffusion of DNA ends after digestion. Interestingly, the number of excess FR reads varies from library to library, which probably reflects differences in the level of chromatin fixation. Thus, excess FR reads should not be used to score the quality of $\mathrm{Hi}-\mathrm{C}$ libraries.

\section{Conclusions}

By comparing and modifying existing methods, we developed a robust and efficient protocol for DNAse I Hi-C analysis of human cells and tissues. We demonstrated the reproducibility of this protocol by applying it to human blood samples. The end-labelling strategy employed in the protocol does not require the incorporation of biotinylated adapters, and DNAse I digestion results in more uniform coverage than restriction enzyme-based approaches. Uniform coverage and the absence of exogenous sequences, which could be erroneously aligned to the reference genome, make this protocol suitable for SNP detection. In addition, the lower noise levels of the protocol developed in this study compared to previously published DNAse I Hi-C protocols should be beneficial for studying the 3D organization of chromatin and detecting chromosomal rearrangements. Thus, the protocol developed in this study could be used in the future to characterize genetic polymorphisms and study chromatin architecture in human cells.

\section{Methods}

\section{Detailed DNase Hi-C protocols}

We describe the protocol "Ramani et al., biotin fill-in". This is the main protocol developed in this study, which allows the preparation of high-quality $\mathrm{Hi}-\mathrm{C}$ libraries without biotinylated adapters. The additional protocols used in this study are described in the Additional file 1. 
1. Cell crosslinking

1.1. Preparation of cell suspensions

A. Peripheral blood samples

Peripheral blood samples were collected in tubes with EDTA. RBC lysis buffer (BioLegend) was used for erythrocyte removal according to the manufacturer's instructions. After lysis, the cell pellets were washed, cells were counted and resuspended in DMEM at a concentration of $1 \times 10^{6}$ cells $/ \mathrm{ml}$.

B. Suspension cells

K562 cells were grown in RPMI-1640 medium with $10 \% \mathrm{FBS}$ and a penicillin/streptomycin mix (all from Invitrogen). The cells were collected, washed to remove traces of serum and resuspended in RPMI-1640 medium at the same concentration.

C. Adherent cells

Adherent LNCap and A549 cells were grown in DMEM with 10\% FBS and a penicillin/ streptomycin mix (all from Invitrogen). The cells were disaggregated by a trypsin (Invitrogen) treatment, washed and resuspended in DMEM at the same concentration.

1.2. Cell fixation

For all cell types, cells were fixed by adding 1\% formaldehyde (Sigma-Aldrich) and incubating for $10 \mathrm{~min}$ at room temperature (RT) with continuous rotation. Crosslinking was quenched by adding $2.5 \mathrm{M}$ glycine to a final concentration of $0.125 \mathrm{mM}$ and incubating for $10 \mathrm{~min}$ at RT with continuous rotation. The cell suspension was centrifuged at $1100 \mathrm{~g}$ for $10 \mathrm{~min}$, resuspended in PBS and split into aliquots of $2.5 \times 10^{6}$ cells. The cells were centrifuged at $1100 \mathrm{~g}$ for another $10 \mathrm{~min}$. The cell pellets were snap-frozen and stored at $-80^{\circ} \mathrm{C}$.

2. Cell lysis

2.1. The pellet of cross-linked cells was placed on ice and gently resuspended in $1 \mathrm{ml}$ of ice-cold cell lysis buffer (10 mM Tris- $\mathrm{HCl} \mathrm{pH} \mathrm{8.0,}$ $10 \mathrm{mM} \mathrm{NaCl}, 0.2 \%$ Igepal).

2.2. The pellet was then incubated on ice for $20 \mathrm{~min}$ with intermittent rotation.

2.3. Centrifugation was performed at $2500 \mathrm{~g}$ for 5 min.

2.4. The supernatant was removed, and the pellet was gently resuspended in $100 \mu \mathrm{l}$ DNase buffer
(50 mM Tris- $\mathrm{HCl} \mathrm{pH} 7.5,0.5 \mathrm{mM} \mathrm{CaCl}_{2}$ ) with $5 \mathrm{mM} \mathrm{MnCl}_{2}$ and $0.2 \%$ SDS.

2.5. The resuspended pellet was incubated at $37^{\circ} \mathrm{C}$ for $30 \mathrm{~min}$.

Control point 1: $5 \mu \mathrm{l}$ lysed cells were saved to check the DNase I digestion efficiency.

2.6. SDS was quenched by adding $100 \mu \mathrm{l}$ DNase buffer $(50 \mathrm{mM}$ Tris- $\mathrm{HCl} \mathrm{pH}$ 7.5, $0.5 \mathrm{mM}$ $\mathrm{CaCl}_{2}$ ) with $5 \mathrm{mM} \mathrm{MnCl}$ and $2 \%$ Triton X-100

2.7. The quenched reaction mixture was incubated at $37^{\circ} \mathrm{C}$ for $10 \mathrm{~min}$.

2.8. DNase I (1.5 U; Thermo Scientific) was added and incubated at RT for 5 min (note: do not incubate mixture longer than $5 \mathrm{~min}$ ).

2.9. The reaction was stopped immediately after 5 min by adding $40 \mu$ Stop buffer $(125 \mathrm{mM}$ EDTA, 2.5\% SDS).

Control point 2: $10 \mu \mathrm{l}$ of the reaction was saved to check the DNase I digestion efficiency. Ninety microlitres of lysis buffer (10 mM Tris- $\mathrm{HCl} \mathrm{pH} \mathrm{8.0,}$ $10 \mathrm{mM} \mathrm{NaCl}, 0.2 \% \mathrm{SDS}$ ) and $5 \mu \mathrm{l}$ proteinase $\mathrm{K}$ (800 units/ml) were added to both controls. The controls were reverse cross-linked at $65^{\circ} \mathrm{C}$ for at least $3 \mathrm{~h}$. DNA was extracted by the standard phenol-chloroform method.

2.10. The reaction was centrifuged at $2500 \mathrm{~g}$ for $5 \mathrm{~min}$.

2.11. The supernatant was discarded, and the pellet was resuspended in $100 \mu \mathrm{l}$ nuclease-free water.

2.12. Two hundred microlitres of AMPure beads were added and mixed well.

2.13. The mixture was incubated for $5 \mathrm{~min}$ at RT, and the tube was exposed to a magnet for $2 \mathrm{~min}$.

2.14. The supernatant was discarded, and the beads were washed twice with freshly prepared $80 \%$ ethanol. The mixture was spun gently (no more than 2500g), and residual ethanol was removed. The beads were air dried for no more than 2 min.

2.15. The beads were resuspended in $100 \mu \mathrm{l}$ NEBuffer 3.1, and the AMPure Beads remained in the mixture.

3. Biotin labelling (volume: $200 \mu \mathrm{l}$ )

3.1. The following components were mixed on ice:

\begin{tabular}{lll}
\hline Reagents & $\begin{array}{l}\text { Amount per tube } \\
(\mu \mathrm{l})\end{array}$ & Final \\
\hline NEBuffer 3.1 & 10 & $1 \mathrm{X}$ \\
dATP, $10 \mathrm{mM}$ & 1.5 & $75 \mu \mathrm{M}$ \\
dTTP, 10 mM & 1.5 & $75 \mu \mathrm{M}$ \\
\hline
\end{tabular}




\begin{tabular}{lll}
\hline Reagents & $\begin{array}{l}\text { Amount per tube } \\
(\mu \mathrm{l})\end{array}$ & Final \\
\hline $\mathrm{dGTP}, 10 \mathrm{mM}$ & 1.5 & $75 \mu \mathrm{M}$ \\
$\begin{array}{l}\text { Biotin-15-dCTP, } \\
\quad 1 \mathrm{mM}\end{array}$ & 15 & $75 \mu \mathrm{M}$ \\
Klenow $(5 \mathrm{U} / \mu \mathrm{l})$ & 10 & $50 \mathrm{U}$ \\
$\mathrm{H}_{2} \mathrm{O}$ & 60.5 & \\
\hline
\end{tabular}

3.2. The above components were added to the tube from step 2.14.

3.3. The mixture was incubated at $23{ }^{\circ} \mathrm{C}$ on a thermomixer for $4 \mathrm{~h}$ with intermittent gentle shaking.

4. In situ ligation (volume $1000 \mu \mathrm{l}$ )

4.1. The following components were mixed on ice:

\begin{tabular}{lll}
\hline Reagents & $\begin{array}{l}\text { Amount per tube } \\
(\mu \mathrm{l})\end{array}$ & Final \\
\hline $10 \times$ T4 ligase buffer & 100 & $1 \mathrm{X}$ \\
$10 \%$ Triton X-100 & 100 & $1 \%$ \\
$25 \%$ PEG-8000 & 200 & $5 \%$ \\
BSA 100 mM & 10 & $1 \mathrm{mM}$ \\
T4 DNA ligase () & 20 & \\
$\mathrm{H}_{2} \mathrm{O}$ & 370 &
\end{tabular}

4.2. The above components were added to the tube from step 3.3.

4.3. The mixture was incubated at $16{ }^{\circ} \mathrm{C}$ on a thermomixer for at least $8 \mathrm{~h}$ (night is also appropriate) with continuous shaking.

5. Cross-link reversal

5.1. Centrifuge the reaction mixture obtained after step 4.3 at $2500 \mathrm{~g}$ for $3 \mathrm{~min}$.

5.2. The supernatant was discarded, and the pellet was resuspended in $400 \mu \mathrm{l}$ NEBuffer 2.

5.3. Twelve microlitres of $10 \%$ SDS was added to the resuspended pellet.

5.4. Twenty microlitres of proteinase K (800 units/ $\mathrm{ml})$ was then added to the mixture.

5.5. The mixture was incubated at $65{ }^{\circ} \mathrm{C}$ on a thermomixer for $4 \mathrm{~h}$ with continuous shaking.

5.6. Twenty microlitres of proteinase K (800 units/ $\mathrm{ml})$ was then added.

5.7. The mixture was incubated at $65{ }^{\circ} \mathrm{C}$ on a thermomixer for $4 \mathrm{~h}$ (this step can be performed at night) with continuous shaking.

5.8. Three microlitres Glycoblue, $50.5 \mu \mathrm{l} 3 \mathrm{M} \mathrm{NaAc}$ and $506 \mu$ isopropanol were then added.

5.9. The mixture was incubated at $-80{ }^{\circ} \mathrm{C}$ for $20 \mathrm{~min}$
5.10. The mixture was then centrifuged at greater than $15,000 \mathrm{~g}$ for $40 \mathrm{~min}$ at $4{ }^{\circ} \mathrm{C}$.

5.11. The supernatant was discarded, and the DNA and AMPure Beads pellet was resuspended with $100 \mu \mathrm{l}$ nuclease-free water containing $5 \mu \mathrm{g}$ RNase A.

5.12. The resuspended pellet was incubated at $37^{\circ} \mathrm{C}$ on a thermomixer for 30 min with continuous shaking.

5.13. Fifty microlitres AMPure beads (0.5x) were added and mixed well.

5.14. The mixture was incubated for $5 \mathrm{~min}$ at RT, and the tube was exposed to a magnet for 2 min.

5.15. The supernatant was discarded, and the beads were washed twice with freshly prepared $80 \%$ ethanol and spun briefly. The residual ethanol was removed. Then, the beads were air dried for no more than $2 \mathrm{~min}$.

5.16. The beads were resuspended in $50 \mu \mathrm{l}$ nucleasefree water.

5.17. The beads were incubated for $10 \mathrm{~min}$ at $\mathrm{RT}$ and then collected via a magnet. The supernatant was transferred to a new $1.5 \mathrm{ml}$ tube.

5.18. The concentration of the recovered DNA was measured with a Qubit fluorometer. The yield was 3-6 $\mu \mathrm{g}$ if starting with $2.5 \times 10^{6}$ cells.

6. Removal of biotin from unligated ends (volume: $100 \mu \mathrm{l})$

6.1. The following components were mixed on ice:

\begin{tabular}{lll}
\hline Reagents & $\begin{array}{l}\text { Amount per } \\
\text { tube }(\mu \mathrm{l})\end{array}$ & Final \\
\hline Purified DNA & 50 & \\
NEBuffer 2.1 & 10 & $1 \mathrm{X}$ \\
dATP, $1 \mathrm{mM}$ & 1.2 & $12 \mu \mathrm{M}$ \\
dGTP, 1 mM & 1.2 & $12 \mu \mathrm{M}$ \\
EDTA, 25 mM & 1 & $0.25 \mathrm{mM}$ \\
T4 DNA polymerase $(3 \mathrm{U} / \mu \mathrm{l})$ & 1.6 & $5 \mathrm{U}$ \\
$\mathrm{H}_{2} \mathrm{O}$ & 35 & \\
\hline
\end{tabular}

6.2. The above components were incubated at $20^{\circ} \mathrm{C}$ in a thermocycler for $1 \mathrm{~h}$ for $30 \mathrm{~min}$.

We strongly recommend testing T4 DNA polymerase before proceeding with the following steps.

6.3. The reaction was stopped by adding $5 \mu \mathrm{l}$ 500 mM EDTA.

6.4. One hundred and five microlitres AMPure beads $(1 \mathrm{x})$ were added and mixed well. 
6.5. The mixture was incubated for $5 \mathrm{~min}$ at RT, and the tube was exposed to a magnet for 2 min.

6.6. The supernatant was discarded, and the beads washed twice with freshly prepared $80 \%$ ethanol and spun briefly. The residual ethanol was removed. Then, the beads were air dried for no more than 2 min.

6.7. The beads in were resuspended in $120 \mu$ l nuclease-free water.

6.8. The beads were incubated for $10 \mathrm{~min}$ at RT and collected via a magnet. The supernatant was transferred to a new $1.5-\mathrm{ml}$ tube.

6.9. The concentration of the recovered DNA was measured with a Qubit fluorometer. It is normal if the amount of DNA is about $30-70 \%$ of original amount after these steps.

\section{Sonication}

7.1. DNA was transferred to a Covaris microtube.

7.2. DNA was sheared to a size of $200-400 \mathrm{bp}$. For Covaris, the following parameters were used: duty factor, 20; peak power, 50; cycles per burst, 200; and time, $110 \mathrm{~s}$

7.3. Sheared DNA was transferred to a new 1.5-ml tube marked as "L".

\section{Size select}

8.1. The volume of sheared DNA (tube "L") was brought to $200 \mu \mathrm{l}$ with nuclease-free water.

8.2. One hundred and twenty-five microlitres of AMPure beads were added and mixed well.

8.3. The mixture was incubated for $10 \mathrm{~min}$ at RT and exposed to a magnet for $2 \mathrm{~min}$.

8.4. Meanwhile, $200 \mu \mathrm{l}$ AMPure beads were added to a new $1.5-\mathrm{ml}$ tube (marked as " $\mathrm{S}$ "), and the tube was exposed to a magnet for $2 \mathrm{~min}$.

8.5. The supernatant was discarded, and $100 \mu \mathrm{l}$ AMPure beads were added and mixed well.

8.6. The supernatant from tube "L" was transferred to tube "S" and mixed well.

8.7. The mixture was incubated for $10 \mathrm{~min}$ at RT, and the tube was exposed to a magnet for 2 min.

8.8. The supernatant was discarded, and the beads were washed twice with freshly prepared 80\% ethanol and spun briefly. Residual ethanol was removed. Then, the beads were air dried for no more than $2 \mathrm{~min}$.
8.9. The beads were resuspended in $50 \mu \mathrm{l}$ nucleasefree water.

8.10. The beards were incubated for $10 \mathrm{~min}$ at RT and collected via a magnet. The supernatant was transferred to a new 1.5 -ml tube.

8.11. The concentration of the recovered DNA was measured with a Qubit fluorometer. DNA amount could be about $50 \%$ of initial amount after these steps.

9. End repair and dA-tailing (volume $60 \mu \mathrm{l}$ ). We used the KAPA HyperPrep Kit.

9.1. One microgram purified DNA was transferred to a new 0.2-ml tube.

9.2. The volume of DNA was brought to $50 \mu \mathrm{l}$ with nuclease-free water.

9.3. The following components were mixed on ice:

\begin{tabular}{ll}
\hline Reagents & $\begin{array}{l}\text { Amount } \\
\text { per tube } \\
(\mu l)\end{array}$ \\
\hline End repair and A-tailing buffer & 7 \\
End repair and A-tailing enzyme mix & 3
\end{tabular}

9.4. The above components were added to the tube from step 9.1.

9.5. The mixture was incubated at $20{ }^{\circ} \mathrm{C}$ for $30 \mathrm{~min}$ and then at $65{ }^{\circ} \mathrm{C}$ for $30 \mathrm{~min}$ in a thermocycler with the lid at $85^{\circ} \mathrm{C}$ to inactivate enzymes.

10. Adapter ligation (volume: $110 \mu \mathrm{l}$ ). We used the KAPA HyperPrep Kit.

10.1 The following components were mixed on ice:

\begin{tabular}{lc}
\hline Reagents & $\begin{array}{l}\text { Amount } \\
\text { per tube } \\
(\mu \mathrm{l})\end{array}$ \\
\hline DNA from the step 9.5 & 60 \\
$\mathrm{H}_{2} \mathrm{O}$ & 5 \\
Adapter stock (concentration as required) & 5 \\
Ligation buffer & 30 \\
DNA Ligase & 10 \\
\hline
\end{tabular}

10.2. The above components were incubated at $20^{\circ} \mathrm{C}$ for $30 \mathrm{~min}$ in a thermocycler with the lid OFF.

10.3. Eighty-eight microlitres AMPure beads were added and mixed well.

10.4. The mixture was incubated for $5 \mathrm{~min}$ at $\mathrm{RT}$, and the tube was exposed to magnet for $2 \mathrm{~min}$. 
10.5. The supernatant was discarded, and the beads were washed twice with freshly prepared $80 \%$ ethanol and spun briefly. Residual ethanol was removed. Then, the beads were air dried for no more than 2 min.

10.6. The beads were resuspended in $100 \mu \mathrm{l}$ nuclease-free water.

10.7. The beads were incubated for $10 \mathrm{~min}$ at RT and were collected via a magnet. The supernatant was transferred to a new 1.5ml tube.

11. Biotin pulldown

11.1. Thirty microlitres Dynabeads ${ }^{\circledR}$ MyOne $^{\mathrm{TM}}$ Streptavidin $\mathrm{C} 1$ and $100 \mu \mathrm{l}$ of $1 \times \mathrm{B} \& \mathrm{~W}$ buffer (5 mM Tris- $\mathrm{HCl}$ pH 8.0, 0.5 mM EDTA, $1 \mathrm{M}$ $\mathrm{NaCl}$ ) were mixed in a 1.5 - $\mathrm{ml}$ low binding tube, and the tube was exposed to a magnet for 2 min.

11.2. The supernatant was discarded, and $100 \mu \mathrm{l}$ of $1 \times \mathrm{B} \& \mathrm{~W}$ buffer was added to the beads and mixed well. The tube was exposed to a magnet for $2 \mathrm{~min}$.

11.3. The supernatant was discarded, and $100 \mu \mathrm{l}$ $2 \times \mathrm{B} \& \mathrm{~W}$ buffer $(10 \mathrm{mM}$ Tris- $\mathrm{HCl} \mathrm{pH} 8.0$, $1 \mathrm{mM}$ EDTA, $2 \mathrm{M} \mathrm{NaCl}$ ) was added.

11.4. Purified adapter-ligated DNA was added to the beads from step 10.7 and mixed well.

11.5. The mixture was incubated for $15 \mathrm{~min}$ at RT with rotation.

11.6. The tube was exposed to a magnet for $2 \mathrm{~min}$, and the supernatant was discarded.

11.7. The beads were washed four times with $200 \mu \mathrm{l}$ $1 \times \mathrm{B} \& \mathrm{~W}$ buffer with the addition $0.1 \%$ Tween20.

11.8. The tube was washed two times with $200 \mu \mathrm{l}$ $10 \mathrm{mM}$ Tris $-\mathrm{HCl}, \mathrm{pH} 8.0$, and before the last wash, the tube was changed.

11.9. The beads were resuspended in $40 \mu \mathrm{l}$ 10 mM Tris-HCl, pH 8.0.

12. Amplification (volume $50 \mu \mathrm{l}$ ). We used the KAPA HyperPrep Kit.

12.1. The following components were mixed on ice:

\begin{tabular}{lll}
\hline Reagents & $\begin{array}{l}\text { Amount per } \\
\text { tube }(\mu l)\end{array}$ & Final \\
\hline DNA-bound streptavidin beads & 20 & \\
KAPA HiFi HotStart ReadyMix (2x) & 25 & $1 \mathrm{x}$ \\
\hline
\end{tabular}

\begin{tabular}{lll}
\hline Reagents & $\begin{array}{l}\text { Amount per } \\
\text { tube }(\mu \mathrm{l})\end{array}$ & Final \\
\hline $\begin{array}{l}\text { KAPA Library Amplification Primer } \\
\text { Mix (10x) }\end{array}$ & 5 & $1 \mathrm{x}$ \\
\hline
\end{tabular}

12.2. The following PCR program was used:

1 cycle-Initial denaturation, $98^{\circ} \mathrm{C}$ for $45 \mathrm{~s}-1$ cycle

0-20 cycle-Denaturation, $98^{\circ} \mathrm{C}$ for $15 \mathrm{~s}$.

Annealing, $60^{\circ} \mathrm{C}$ for $30 \mathrm{~s}$.

Extension, $72{ }^{\circ} \mathrm{C}$ for $30 \mathrm{~s}$.

1 cycle - Final extension, $72{ }^{\circ} \mathrm{C}$ for $1 \mathrm{~min}$

$\operatorname{HOLD} 4{ }^{\circ} \mathrm{C} \infty$.

12.3. Fifty microlitres AMPure beads were added to the reaction mix and mixed well.

12.4. The mixture was incubated for $5 \mathrm{~min}$ at RT, and the tube was exposed to a magnet for 2 min.

12.5. The supernatant was discarded, and the beads were washed twice with freshly prepared $80 \%$ ethanol and spin briefly. Residual ethanol was removed. Next, the beads were air dried for no more than $2 \mathrm{~min}$.

12.6. The beads were resuspended in $40 \mu \mathrm{l}$ nucleasefree water.

12.7. The beads were incubated for $10 \mathrm{~min}$ at $\mathrm{RT}$ and were collected via a magnet. The supernatant was transferred to a new 0.5 -ml tube.

12.8. The concentration of the recovered DNA was measured with a Qubit fluorometer.

13. Check the quality.

13.1 Two nanograms of the amplified library was analysed using an Agilent High Sensitivity DNA Kit according to the manufacturer's instructions. The library displayed a fragment size distribution in the range of 150 to $500 \mathrm{bp}$.

\section{Computational analysis of DNase Hi-C libraries Mapping and processing of sequence reads}

We sequenced the targeted DNase Hi-C libraries using paired-end reads with a length of $150 \mathrm{bp}$. Next, we mapped the paired-end reads to the human hg19 genome using BWA-MEM [27] with the default parameters. We did not remove the Illumina adapters before mapping and decided to not split the reads by ligation junctions because bwa could successfully map the reads (Additional file 1: Table S2, Fig. 3). To define interacting genomic fragments, we searched for the greatest distance between the coordinates of all the primary and supplementary alignments from both read mates. To obtain valid interaction pairs, we only included reads with both mates mapped uniquely $($ mapq $>0)$. We removed PCR 
duplicates from the Hi-C data: we defined two read pairs as duplicates if they shared the alignment position of both mates. Unique read filtering and duplicate removal were performed using an in-house developed pipeline. The scripts that we used for $\mathrm{Hi}-\mathrm{C}$ data processing and quality analysis are available on GitHub https://github. com/evgeniy240294/ExoC.

As an alternative to bwa, we used bowtie2 [29] wrapped in the Hi-C Pro [28] pipeline. We used $\mathrm{Hi}-\mathrm{C}$ Pro with the default parameters. We provided the sequence of the bridge adapter as a ligation junction sequence. To remove the adapter sequences from the read ends, we used cutadapt [30] iteratively in the noninternal adapter mode with the following parameters: minimum overlap equal to 5 and minimum length equal to 7 . We searched for all the variants of the multimers in every iteration. We performed five consecutive iterations of cutadapt processing to remove adapter concatemers.

\section{Quality control of $\mathrm{Hi}-\mathrm{C}$ data}

To evaluate the number of dangling ends (DE), we used the following equation:

$$
\mathrm{DE}=(F R+R F)-(F F+R R),
$$

where $F R, R F, F F$, and $R R$ are the number of valid pairs with read mates in the forward-reverse, reverse-forward, forward-forward and reverse-reverse orientations, respectively. It was assumed that the $F R, R F, F F$ and $R R$ classes of the $\mathrm{Hi}-\mathrm{C}$ read orientations were distributed at a ratio of 1:1:1:1 and that overrepresentations of $F R$ or $R F$ might indicate the presence of either nonligated fragments or back ligations, respectively.

To evaluate the number of back ligations $(B)$ in $\mathrm{DE}$, we used datasets prepared using a biotinylated oligonucleotide adapter. This allowed the identification of a fraction of reads that had bridge adapters in their sequence. First, we used cutadapt as described above to remove adapters from the $5^{\prime}$-ends of reads, thus keeping only reads containing noninternal adapter sequences. Notably, the read length was $150 \mathrm{bp}$; therefore, the adapter sequence could be undetected if the DNA insert was more than $300 \mathrm{bp}$. Therefore, we only considered reads that were less than $300 \mathrm{bp}$. For this purpose, we used the AdapterRemoval tool [31] without specifying any adapter sequence and with the default parameters. In this mode, the tool allowed us to find all the reads that had two mate sequences overlapping with each other, which means that they represented DNA fragments with inserts of less than 300 bp completely covered by two mates with a length of $150 \mathrm{bp}$.

The presence of an adapter within a read sequence could indicate either that the adapter was ligated to the DNA end during Hi-C library preparation or that the genomic sequence matched the adapter sequence by chance. Thus, if we considered all the read pairs in the FF or RR orientation containing adapter sequences, we could describe them as the sum of adapter ligation events and incidental matches between the adapter sequence and genomic DNA. Formally, we define:

$$
P 1=N \cdot(y+c),
$$

where $\mathrm{P} 1$ is the number of reads in the FF and RR orientations containing the adapter sequence, $\mathrm{N}$ is the total number of FF and RR reads, $y$ is the frequency of adapter ligation and $c$ is the frequency of the incidental occurrence of the adapter sequence within the genome. To compute $c$, we searched for the adapter sequences in the libraries prepared without an adapter (in this case, $y=0$ ) and found that $c \approx 0.05$ for the 7-bp bridge adapter and $c \approx 0$ for a 19-bp long BAT-Hi-C adapter.

Next, we considered the reads in the FR and RF orientations containing the adapter sequence. Without preference for back-ligation and in the absence of undigested DNA, the number of such reads would be similar to the number of FF and RR reads, which is equal to $N \cdot(y+c)$. The back-ligation events expected for equal $\mathrm{FF} / \mathrm{RR} / \mathrm{RF} /$ RR orientations would add to this number $B \cdot(y+c)$, where $B$ is the number of back-ligation events. Reads originating from undigested/unligated DNA could contain the adapter sequence only when it incidentally matched the genomic sequence, which would add $G \cdot c$ reads in the FR or RF orientation with adapter, where $G$ is the number of sequenced undigested/unligated DNA fragments. In total, the number of reads in the FR and RF orientations containing the adapter sequence, which we defined as P2, can be defined as

$$
\begin{aligned}
P 2= & N \cdot(y+c)+G \cdot c+B \cdot(y+c) \\
& =N \cdot(y+c)+(G+B) \cdot c+B \cdot y .
\end{aligned}
$$

In this equation, it is pertinent to note that $(G+B)$ is the sum of back-ligation events and undigested/unligated DNA, which we have previously estimated as DE, i.e. $(G+B)=D E$. We also know that $P 1=N \cdot(y+c)$; therefore,

$$
\begin{gathered}
P 2=P 1+\mathrm{DE} \cdot c+B \cdot y, \text { and } \\
y=\frac{P 1}{N}-c .
\end{gathered}
$$

This allows us to compute $B$ as follows:

$$
B=\frac{P 2-P 1-D E \cdot c}{\frac{P 1}{N}-c} .
$$

Using this equation, we computed $B$ for all the libraries prepared with biotinylated oligonucleotide adapters. 


\section{Supplementary Information}

The online version contains supplementary material available at https://doi. org/10.1186/s13072-021-00389-5.

Additional file 1. Supplementary information: supplementary tables and figures.

\section{Acknowledgements}

We are grateful to all the members of the molecular mechanisms of the development group for the fruitful discussions. We thank the participants of this study who provided the blood samples used in this work.

\section{Authors' contributions}

VF designed and supervised the study; M.G. performed all the wet-lab experiments; EM performed the computational data analysis; EV developed the FastContext algorithms; LPN, MEL, ZGM, MIJ, VJV, NVS and INL provided the human blood samples; VF, MG, EM. and EV wrote the manuscript; all the authors revised the manuscript. All authors read and approved and final manuscript.

\section{Funding}

DNAse I Hi-C library preparation and sequencing were supported by RFBR Grant 18-29-13021. Data analysis performed by E.M. was supported by RFBR Grant 20-34-90110. High-throughput computations were performed on the nodes of the computational cluster of the Institute of Cytology and Genetics SB RAS (budget project no. 0259-2021-0016).

\section{Availability of data and materials}

Sequencing data are accessible via the NCBI GEO database. The scripts used for data processing and analysis are available on GitHub: https://github.com/ evgeniy240294/ExoC and https://github.com/regnveig/labjournal/tree/ master/tools/FastContext.

\section{Declarations}

\section{Ethics approval and consent to participate}

This study was performed in line with the principles of the Declaration of Helsinki. Approval was granted by the local Research Ethics Committee of the Research Institute of Medical Genetics, Tomsk National Research Medical Center (Date 27.07.2017/No 106). All the study participants provided informed consent.

\section{Consent for publication}

Not applicable.

\section{Competing interests}

The authors declare that they have no competing interests.

\begin{abstract}
Author details
${ }^{1}$ Institute of Cytology and Genetics SB RAS, Lavrentjeva ave 10, Novosibirsk, Russia. ${ }^{2}$ Novosibirsk State University, Pirogova str., 2, Novosibirsk, Russia.

${ }^{3}$ Research Institute of Medical Genetics, Tomsk National Research Medical Center, Kooperativny Str, 5, Tomsk, Russia. ${ }^{4}$ Clinical Research Institute of Pediatrics Named After Acad. Y.E. Veltischev, Moscow, Russia. ${ }^{5}$ Research Centre for Medical Genetics, Moskvorechie str., 1, Moscow, Russia.
\end{abstract}

Received: 1 November 2020 Accepted: 8 March 2021 Published online: 20 March 2021

\section{References}

1. Lieberman-Aiden E, van Berkum NL, Williams L, Imakaev M, Ragoczy T, Telling A, et al. Comprehensive mapping of long-range interactions reveals folding principles of the human genome. Science. 2009;326:289-93.

2. Rao SSP, Huntley MH, Durand NC, Stamenova EK, Bochkov ID, Robinson JT, et al. A 3D Map of the Human Genome at Kilobase Resolution Reveals Principles of Chromatin Looping. Cell. 2014;159:1665-1680. http://linki
nghub.elsevier.com/retrieve/pii/S0092867414014974. Accessed 11 Dec 2014.

3. Fishman V, Battulin N, Nuriddinov M, Maslova A, Zlotina A, Strunov A, et al. 3D organization of chicken genome demonstrates evolutionary conservation of topologically associated domains and highlights unique architecture of erythrocytes' chromatin. Nucleic Acids Res Engl. 2019;47:648-65.

4. Battulin N, Fishman VS, Mazur AM, Pomaznoy M, Khabarova AA, Afonnikov DA, et al. Comparison of the 3D organization of sperm and fibroblast genomes using the Hi-C approach. Genome Biol. 2015;16:77. http:// genomebiology.com/2015/16/1/77. Accessed 19 Apr 2015

5. Flyamer IM, Gassler J, Imakaev M, Brandão HB, Ulianov S V., Abdennur $\mathrm{N}$, et al. Single-nucleus $\mathrm{Hi}-\mathrm{C}$ reveals unique chromatin reorganization at oocyte-to-zygote transition. Nature. 2017;544:110-4. https://doi.org/10. 1038/nature21711

6. Burton JN, Adey A, Patwardhan RP, Qiu R, Kitzman JO, Shendure J. Chromosome-scale scaffolding of de novo genome assemblies based on chromatin interactions. Nat Biotechnol. 2013;31:1119-25.

7. Kaplan N, Dekker J. High-throughput genome scaffolding from in vivo DNA interaction frequency. Nat Biotechnol United States. 2013;31:1143-7.

8. Marie-Nelly H, Marbouty M, Cournac A, Flot JF, Liti G, Parodi DP, et al. High-quality genome (re)assembly using chromosomal contact data. Nat Commun. 2014;5(1):1.

9. Dudchenko O, Batra SS, Omer AD, Nyquist SK, Hoeger M, Durand NC, et al. De novo assembly of the Aedes aegypti genome using $\mathrm{Hi}-\mathrm{C}$ yields chromosome-length scaffolds. Science. 2017;356:92-5.

10. Putnam NH, O'Connell BL, Stites JC, Rice BJ, Blanchette M, Calef R, et al. Chromosome-scale shotgun assembly using an in vitro method for longrange linkage. Genome Res. 2016;6(3):342-50.

11. Ghurye J, Rhie A, Walenz BP, Schmitt A, Selvaraj S, Pop M, et al. Integrating $\mathrm{Hi}-\mathrm{C}$ links with assembly graphs for chromosome-scale assembly. PLoS Comput Biol. 2019;15(8):e1007273.

12. Harewood L, Kishore K, Eldridge MD, Wingett S, Pearson D, Schoenfelder $\mathrm{S}$, et al. $\mathrm{Hi}-\mathrm{C}$ as a tool for precise detection and characterisation of chromosomal rearrangements and copy number variation in human tumours. Genome Biol. 2017;8(1):1-1.

13. Wang S, Lee S, Chu C, Jain D, Kerpedjiev P, Nelson GM, et al. HiNT: A computational method for detecting copy number variations and translocations from Hi-C data. Genome Biol. 2020;1(1):1-5.

14. Melo US, Schöpflin R, Acuna-Hidalgo R, Mensah MA, Fischer-Zirnsak $B$, Holtgrewe $\mathrm{M}$, et al. Hi-C Identifies complex genomic rearrangements and TAD-shuffling in developmental diseases. Am J Hum Genet. 2020;106(6):872-84.

15. Díaz N, Kruse K, Erdmann T, Staiger AM, Ott G, Lenz G, et al. Chromatin conformation analysis of primary patient tissue using a low input $\mathrm{Hi}-\mathrm{C}$ method. Nat Commun. 2018;9(1):1-3.

16. Chakraborty A, Ay F. Identification of copy number variations and translocations in cancer cells from Hi-C data. Bioinformatics. 2018;34:338-45.

17. Troll CJ, Putnam NH, Hartley PD, Rice B, Blanchette M, Siddiqui S, et al. Structural variation detection by proximity ligation from formalin-fixed. Paraffin-Embed Tumor Tissue J Mol Diagn. 2019;21(3):375-83.

18. Bansal V. Integrating read-based and population-based phasing for dense and accurate haplotyping of individual genomes. Bioinformatics. 2019.

19. Selvaraj S, Dixon JR, Bansal V, Ren B. Whole-genome haplotype reconstruction using proximity-ligation and shotgun sequencing. Nat Biotechnol. 2013;31(12):1111-8.

20. Mozheiko EA, Fishman VS. Detection of point mutations and chromosomal translocations based on massive parallel sequencing of enriched 3C libraries. Russ J Genet. 2019;55(10):1273-81.

21. Ramani V, Cusanovich DA, Hause RJ, Ma W, Qiu R, Deng X, et al. Mapping 3D genome architecture through in situ DNase Hi-C. Nat Protoc. 2016;11(11):2104-21.

22. Ma W, Ay F, Lee C, Gulsoy G, Deng X, Cook S, et al. Using DNase Hi-C techniques to map global and local three-dimensional genome architecture at high resolution. Methods. 2018;142:59-73.

23. Ma W, Ay F, Lee C, Gulsoy G, Deng X, Cook S, et al. Fine-scale chromatin interaction maps reveal the cis-regulatory landscape of human lincRNA genes. Nat Methods. 2014;12(1):71-8. 
24. Hsieh THS, Weiner A, Lajoie B, Dekker J, Friedman N, Rando OJ. Mapping nucleosome resolution chromosome folding in yeast by micro-C. Cell. 2015;162:108-19.

25. Krietenstein N, Abraham S, Venev SV, Abdennur N, Gibcus J, Hsieh THS, et al. Ultrastructural details of mammalian chromosome architecture. Mol Cell. 2020;8(3):554-65.

26. Durand NC, Shamim MS, Machol I, Rao SSP, Huntley MH, Lander ES, et al. Juicer Provides a One-Click System for Analyzing Loop-Resolution Hi-C Experiments. Cell Syst. Cell Press; 2016;3:95-8. https://inkinghub.elsevier. com/retrieve/pii/S2405471216302198. Accessed 22 Nov 2019.

27. Li H, Durbin R. Fast and accurate short read alignment with BurrowsWheeler transform. Bioinformatics. 2009;25(14):1754-60.

28. Servant N, Varoquaux N, Lajoie BR, Viara E, Chen C-J, Vert J-P, et al. HiC-Pro: an optimized and flexible pipeline for $\mathrm{Hi}-\mathrm{C}$ data processing. Genome Biol [Internet]. 2015;16:259. https://doi.org/10.1186/s13059-015-0831-x

29. Langmead B, Salzberg SL. Fast gapped-read alignment with Bowtie 2. Nat Methods. 2012;9:357-9.
30. Martin M. Cutadapt removes adapter sequences from high-throughput sequencing reads. EMBnet.journal. 2011;17(1):10-2

31. Schubert M, Lindgreen S, Orlando L. AdapterRemoval v2: Rapid adapter trimming, identification, and read merging. BMC Res Notes. 2016;9(1):1-7.

32. Campbell VW, Jackson DA. The effect of divalent cations on the mode of action of DNase I. J Biol Chem. 1980;255(8):3726-35.

33. Suck D, Lahm A, Oefner C. Structure refined to $2 \AA$ of a nicked DNA octanucleotide complex with DNase I. Nature. 1988;332(6163):464-8.

34. Zorbas H, Föckler R, Ernst-Ludwig W. Klenow polymerase is preferable to T4 polymerase in end-labeling reactions. Nucleic Acids Res. 1990;18(19):5909.

\section{Publisher's Note}

Springer Nature remains neutral with regard to jurisdictional claims in published maps and institutional affiliations.
Ready to submit your research? Choose BMC and benefit from:

- fast, convenient online submission

- thorough peer review by experienced researchers in your field

- rapid publication on acceptance

- support for research data, including large and complex data types

- gold Open Access which fosters wider collaboration and increased citations

- maximum visibility for your research: over 100M website views per year

At BMC, research is always in progress.

Learn more biomedcentral.com/submissions 